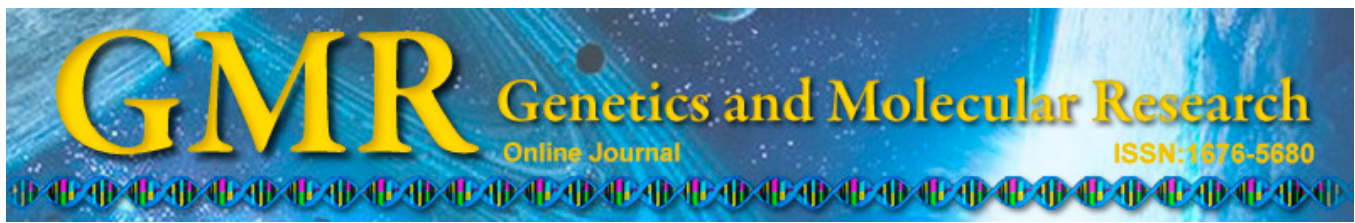

\title{
Transcriptome analysis of Arabidopsis seedlings responses to high concentrations of glucose
}

\author{
L. Han ${ }^{1,2}$, J.L. Li ${ }^{1,3}$, M. Jin ${ }^{1,2}$ and Y.H. Su ${ }^{1}$
}

${ }^{1}$ State Key Laboratory of Soil and Sustainable Agriculture, Institute of Soil Science, Chinese Academy of Sciences, Nanjing, China ${ }^{2}$ Graduate School of Chinese Academy of Science, Beijing, China

${ }^{3}$ College of Forest Resources and Environment, Nanjing Forestry University, Nanjing, China

Corresponding author: Y.H. Su

E-mail: yhsu@issas.ac.cn

Genet. Mol. Res. 14 (2): 4784-4801 (2015)

Received November 4, 2014

Accepted March 10, 2015

Published May 11, 2015

DOI http://dx.doi.org/10.4238/2015.May.11.11

\begin{abstract}
Sugars acting as fuel energy or as signaling molecules play important roles in plant growth and development. Although sugars associated with early seedling development have been analyzed in detail, few studies have examined the effect of sugar on genome-wide gene transcription. To analyze the role of glucose on the genomic level, we examined the response of seedlings to $5 \%$ glucose using RNA-seq technology. High concentrations of glucose significately altered the expression of 863 genes, with 558 upregulated and 305 downregulated genes by more than 2 -fold. A large number of genes affected by glucose were involved in metabolic processes and signaling. Transcript levels for many kinases and calcium signals were downregulated. Most transcription factors identified were also involved in glucose signaling. Moreover, many genes related to the auxin, gibberellin, and abscisic acid responses were upregulated or downregulated. Additionally, the $\mathrm{K}^{+}, \mathrm{Ca}^{2+}, \mathrm{SO}_{3}^{-}, \mathrm{NO}_{3}^{-}, \mathrm{PO}_{4}^{3-}$, amino acid, and sugar transporters were also upregulated or downregulated. These results provide a basic
\end{abstract}


understanding of the glucose-mediated molecular mechanisms in the regulation of early seedling development.

Key words: Arabidopsis; Gene expression; Glucose; Transcriptome

\section{INTRODUCTION}

Plants are capable of monitoring available nutrients and adapting their transcriptional responses and metabolic processes to this information. Sugars, acting as fuel energy or signaling molecules, play important roles in plant growth and development (Smeekens, 2000). Sucrose, glucose, and fructose are the primary sugars in plants. Sucrose is the main form of transported sugars in plants, which is cleaved into fructose and glucose by invertases or stored in vacuoles (Koch, 2004). High concentrations of exogenous glucose were found to suppress hypocotyls elongation, cotyledon greening, and expansion and root development during early seedling development in Arabidopsis (Rolland et al., 2006).

Over the past few decades, several Arabidopsis mutants related to sugar signaling have been isolated and characterized on the molecular level (Smeekens, 2000; León and Sheen, 2003; Rolland et al., 2006). Some of the glucose-insensitive (gin) mutants, which can grow in medium containing $6 \%(\mathrm{w} / \mathrm{v})$ glucose, are allelic to abscisic acid signaling or biosynthesis mutants, indicating that plant glucose signaling interacts with the abscisic acid (ABA) network (León and Sheen, 2003; Rolland et al., 2006). Glucose-insensitive mutant gin5 and gin6/sucrose uncoupling 6/sugar-insensitive 5 are as allelic toaba3 and abi4 (León and Sheen, 2003; Gibson, 2005). The sugar-insensitive 1 as mutant alleles of $c t r l$, a previously isolated mutant showing a constitutive response to ethylene, exhibits resistance to glucose (Gibson et al., 2001). Moreover, the gin 2 mutant is allelic to hexokinase, which catalyzes the conversion of glucose to glucose6-phosphate to overcome growth arrest when glucose concentration is high (Moore et al., 2003).

Auxin is characterized as a root-forming hormone in plants and has been found to have an important role in regulating root growth and development (Overvoorde et al., 2010). Thus, the auxin and glucose may functions may be coordinated during plant root growth and development. The mutant gin 2 was reported to be resistant to exogenous application of auxin (Rolland et al., 2002; Moore et al., 2003). The hookless mutant hls 1 was reported to be resistant to auxin and the sugar response, revealing a strong correlation between auxin and sugar signaling (Ohto et al., 2006). Glucose can also alter auxin transporter PIN proteins, receptor transporter-inhibitor-resistant 1, auxin biosynthetic genes such as YUCCA, and other gene families involved in auxin signaling, including auxin/indole-3-acetic acid (IAA), Gretchenhagen-3 (GH3), and small auxin-up RNA (Mishra et al., 2009).

$\mathrm{Ca}^{2+}$ is a second messenger in many signaling pathways in response to various environmental and developmental stimuli in plants. A large number of $\mathrm{Ca}^{2+}$ sensors, including calmodulins, calcineurin B-like proteins (CBLs), calcium-dependent protein kinases, and calmodulin-like proteins, have been identified in plants (Kudla et al., 2010). The CBL and CBL-interacting protein kinase (CIPK) proteins are specific in plants and were first identified in Arabidopsis (Shi et al., 1999). In Arabidopsis, a total of 10 CBLs and 26 CIPKs have been identified (Weinl and Kudla, 2009). The CBL-CIPK network has been reported to participate in modulating the $\mathrm{Na}^{+}, \mathrm{K}^{+}$, and $\mathrm{NO}_{3}{ }^{-}$transportation (Liu et al., 2000; Xu et al., 2006). Recent studies demonstrated that CBL1 and CIPK14 play an important role in glucose response and signaling (Li et al., 2013; Yan et al., 2014). 
In this study, a high glucose level was shown to markedly reduce primary root length by reducing the length of the root meristematic zone and elongation and mature cells. We used RNA-seq to examine the transcriptomic profiles of Arabidopsis seedlings in response to high concentrations of glucose. We analyzed the functional categorization of differentially expressed genes on a genome-wide level in Arabidopsis. These results provide a basic understanding of the glucose-mediated molecular mechanisms regulating early seedling development.

\section{MATERIAL AND METHODS}

\section{Plant growth conditions}

Seeds of Arabidopsis thaliana (Col-0) were surface-sterilized, rinsed thoroughly, germinated on nutrient plates containing 1/4 strength Murashige and Skoog (MS) medium solidified with $0.8 \%$ agar and supplemented with $1 \%(\mathrm{w} / \mathrm{v})$ glucose as the control condition $(\mathrm{CK})$ or $5 \%$ glucose treatment (Glu), and allowed to grow for 6 days at $23^{\circ} \mathrm{C}$ under a 16 -h light $/ 8$-h dark photoperiod at $100 \mu \mathrm{Em}^{-2} \mathrm{sec}^{-1}$. Samples of CK and Glu were used for phenotype analysis and RNA-seq experiments.

\section{Measurement of root length}

Digital images of 6-day-old seedlings under normal conditions or glucose treatment were captured. The seedlings were washed twice with Mili-Q water, placed on glass slides, and observed under a Nikon microscope (Tokyo, Japan). The primary root length, meristematic zone, elongation zone, and mature cells were quantified using the Image $\mathrm{J}$ software. Error bars indicated SD based on the measurement of at least 40 seedlings per treatment. More than 3 biological replicates were performed for each treatment.

\section{Measurement of anthocyanin}

Anthocyanin contents of whole seedlings were determined using the method described by Das et al. (2012). Absorbance (A) of the supernatant extract was measured at 530 and 657 $\mathrm{nm}$, and the concentration of anthocyanin was calculated as A530-0.25A657.

\section{RNA extraction, library construction, and sequencing}

After glucose treatment, total RNA was isolated and purified using Trizol (Invitrogen, Carlsbad, CA, USA) according to the manufacturer manual. The constructed pair-end libraries were sequenced using an Illumina HiSeqTM 2000 (Shanghai Personal Biotechnology Co. Ltd., Shanghai, China). Raw reads produced from sequencing machines with adaptors, length shorter than 50 base pairs, without uncertainty nucleotides, and of low quality $(<$ Q20) were discarded.

\section{Mapping to genomes and functional annotation}

After trimming and quality evaluation, we used bowtie2/tophat2 (http://tophat.cbcb. umd.edu/) to map the useful reads to Arabidopsis_thaliana.TAIR10.20, the reference genomes, and obtained the mapped reads. Using BLASTx alignment between the genes and the databas- 
es Ensembl, eggnog, and Kyoto Encyclopedia of Genes and Genomes database, we obtained functional annotations and metabolic pathway annotations. Gene Ontology (GO) classification of all genes displaying significant changes in transcript abundance was performed using the AgriGO online tool (Du et al., 2010).

\section{Differential expression analysis of genes}

To identify the differential expression of unigenes affected by glucose, we used the reads per kb per million reads to calculate the expression levels of unigenes. Equal or greater than 2-fold changes was used to identify differentially expressed genes between glucose-treated samples and control samples, with $\mathrm{P}$ value $<0.05$.

\section{Validation of RNA-seq data using real-time-polymerase chain reaction (PCR)}

A total of 10 genes were selected for validation of RNA-seq data using real-time PCR. From the normal condition and glucose treatment samples, $1.5 \mu \mathrm{g}$ were reverse-transcribed into single-strand cDNAs using Oligo- $\mathrm{dT}_{18}$ primer and reverse transcriptase (Takara, Shiga, Japan) according to the manufacturer instruction. For real-time quantitative reverse transcription-PCR, amplification was performed in a $20-\mu \mathrm{L}$ reaction system containing $2.5 \mu \mathrm{L} 5 \mathrm{X}$ diluted cDNA templates, $0.2 \mu \mathrm{M}$ of each forward and reverse primers, and $10 \mu \mathrm{LSYBR}$ Green PCR Master Mix (Toyobo, Osaka, Japan). The reaction was performed in a CFX96TM Real-Time PCR cycler using the following program: 45 cycles at $95^{\circ} \mathrm{C}$ for $15 \mathrm{~s}, 60^{\circ} \mathrm{C}$ for $15 \mathrm{~s}$, and $72^{\circ} \mathrm{C}$ for 15 s. AtActin2 (AT3G18780) was used as an internal control. Primers for each selected gene are presented in Table S1.

\section{RESULTS}

\section{High concentrations of glucose inhibits early seedlings development of Arabidopsis}

Plants are capable of monitoring the nutrients available and adapting their transcriptional responses and metabolic processes to this information. Sugars, acting as fuel energy or signaling molecules, play important roles in plant growth and development (Smeekens, 2000). To examine the transcriptional changes in Arabidopsis at high glucose concentration, wild-type Col-0 were grown on medium supplied with $1 \%$ glucose (w/v) as CK or Glu for 6 days respectively. The effect of glucose on the phenotypes of Arabidopsis seedlings was then evaluated.

The primary roots of Col- 0 seedlings were significantly inhibited by $5 \%$ glucose as shown in Figure 1a and b. To investigate this inhibition in detail, we determined the length of root elongation and meristematic zone as well as mature cells from glucose-treated seedlings (Figure 1c and d). The elongation and meristematic zones of roots treated with $5 \%$ glucose were 346 and $251 \mu \mathrm{m}$ respectively, compared with 492 and $332 \mu \mathrm{m}$ under control condition (Figure 1c and d). In addition, the length of cells in the mature zone was significantly decreased by high glucose concentrations (Figure 1e). Additionally, the leaves of glucose-treated seedlings were less green than those of CK. We then analyzed anthocyanin accumulation in whole seedlings. As shown in Figure 1f, anthocyanin accumulation in seedlings under high concentrations of glucose treatment was increased by approximately 4-fold compared with that in seedlings under control conditions. 

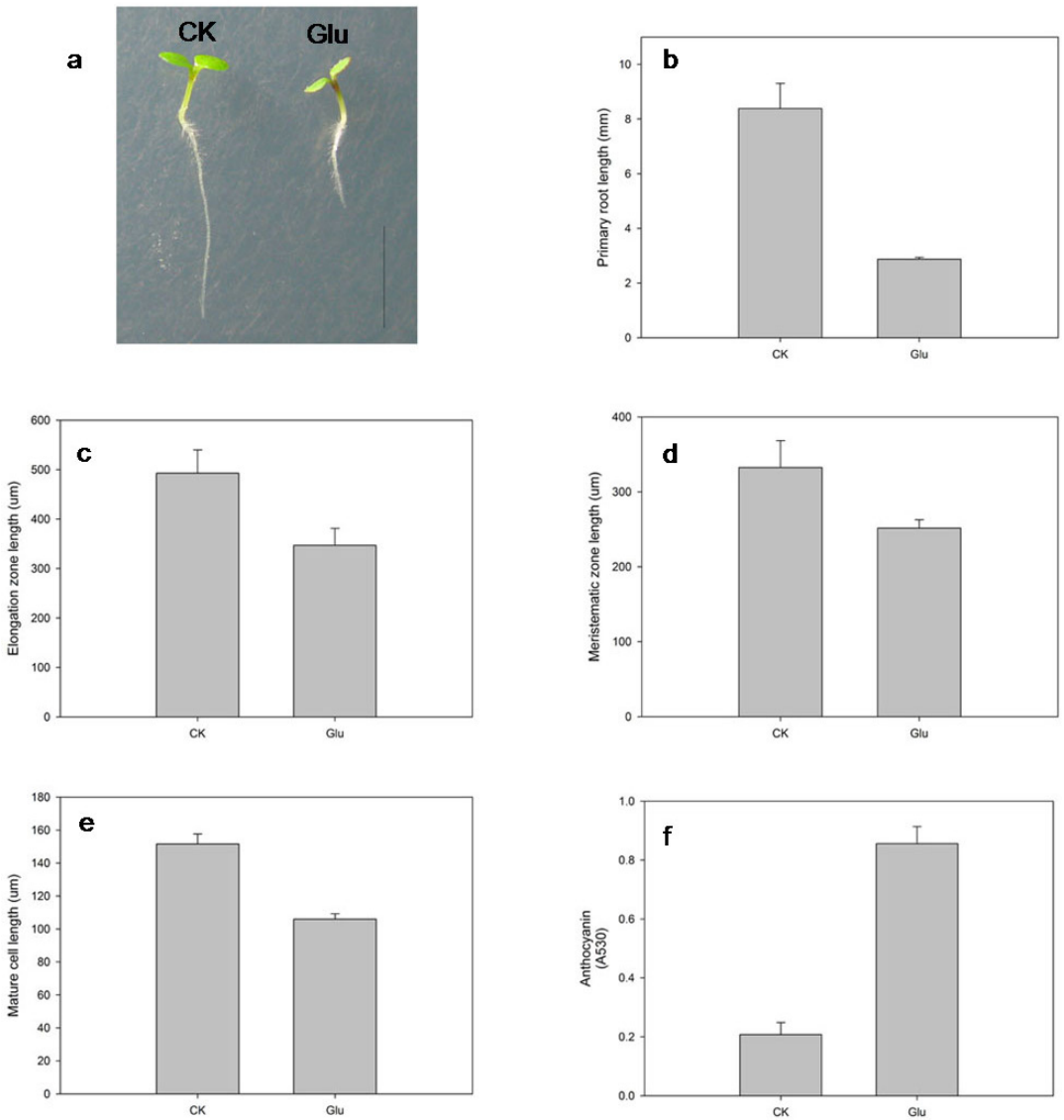

Figure 1. High concentrations of glucose inhibited early seeding development. Wild-type Col-0 were grown on $1 / 4$ MS medium supplied with $1 \%$ glucose $(\mathrm{w} / \mathrm{v})$ as the control condition $(\mathrm{CK})$ or $1 / 4 \mathrm{MS}$ medium with $5 \%$ glucose $(\mathrm{w} / \mathrm{v})$ as the treatment condition (Glu) for 6 days. (a) Phenotype comparison of Arabidopsis seedlings treated with glucose. Bar $=0.5 \mathrm{~cm}$. Length of primary root $(\mathbf{b})$, elongation zone $(\mathbf{c})$, meristematic zone (d), and mature cells $(\mathbf{e})$. (f) Anthocyanin accumulation in Arabidopsis seedlings. Error bars represent the mean SD of 3 independent experiments.

\section{RNA-seq analysis revealed Arabidopsis genes showing transcriptional changes in response to high glucose concentrations}

We used RNA-seq to investigate the genome-wide transcriptional response to high concentrations of glucose; 2 seedling samples treated with 1or 5\% glucose were sequenced using an Illumina HiSeq ${ }^{\mathrm{TM}}$ 2000. We obtained approximately 63 million high-quality reads of 130 base pairs from 2 samples. After quality evaluation and trimming, more than $97 \%$ reads could be mapped back to the Arabidopsis TAIR 10.20 genomes (Table 1). We detected 27,206 genes using RNA-seq, and read counts were normalized and differentially expressed genes (DEGs) were identified in the 2 conditions using the HTSeq and DESeq package (Table S2).DEGs were defined to have 2 -fold or great changes with a false discovery rate-adjusted $\mathrm{P}$ value $<0.05$. Compared to the normal condition, 863 DEGs were identified at high concentrations of glucose. Among these DEGs, 558 genes were upregulated and 305 genes were downregulated by $5 \%$ glucose treatment. 
Table 1. Mapping results of RNA-seq reads from control and glucose treatments in Arabidopsis.

\begin{tabular}{lccc}
\hline Sample & Useful reads & Mapped reads & Mapped percent \% \\
\hline CK & $34,121,162$ & $33,392,312$ & $97.86 \%$ \\
Glu & $29,134,501$ & $28,563,185$ & $98.04 \%$ \\
\hline
\end{tabular}

Gene ontology (GO) analysis of differentially expressed genes in Arabidopsis response to high glucose concentrations

To investigate the underlying functions of DEGs in response to high concentrations of glucose, GO analysis was examined using the AgriGO online tool (Du et al., 2010). The AgriGO service assigned general parent terms to the genes according to biological process, molecular function, and cellular component. The DEGs were classified into 24 biological processes mainly including metabolic process, cellular process, response to stress, transport, response to endogenous stimulus, response to external stimulus, and response to abiotic stimulus, as shown in Table 2. Further evaluation of DEGs in the metabolic process category indicated that approximately $23 \%$ were involved in nitrogen metabolism, $20 \%$ in secondary metabolism process, $17 \%$ in lip metabolism, $16 \%$ in carbohydrate metabolism, and $10 \%$ in phosphorus metabolic process, together accounting for approximately $86 \%$ of genes in this category (Figure $2 a$ ).

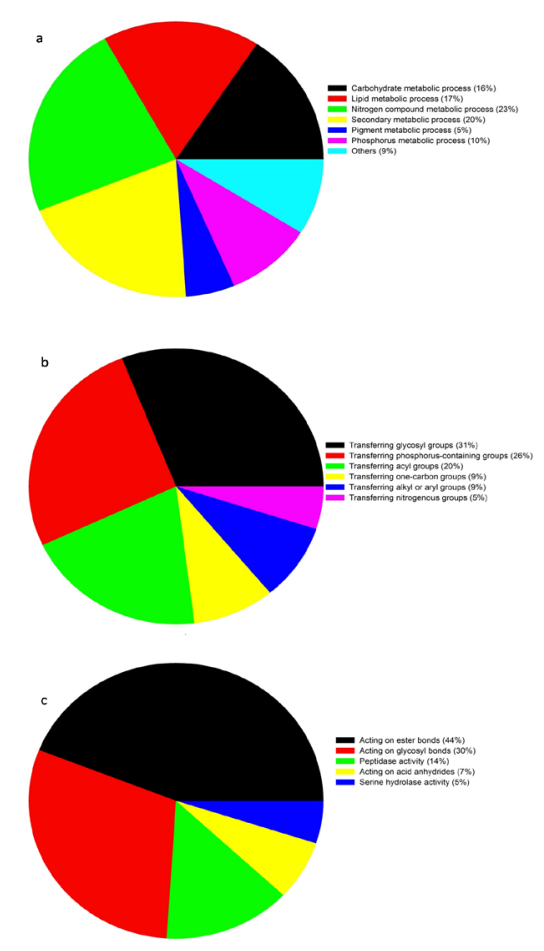

Figure 2. Functional classifications of genes exhibit transcriptional changes in 3 categories. Classification of genes exhibiting transcriptional changes in the categories of metabolic processes (a), transferase activity (b), and hydrolase activity (c) were performed using the AgriGO web service. 
Table 2. Gene ontology classification into biological process of all genes significantly $(\mathrm{P}<0.05)$ differentially expressed in response to $5 \%$ glucose.

\begin{tabular}{|c|c|c|c|c|c|}
\hline \multirow[t]{2}{*}{ Description } & \multirow[t]{2}{*}{ GO } & \multicolumn{2}{|c|}{ Downregulated } & \multicolumn{2}{|c|}{ Upregulated } \\
\hline & & Count & Percent $(\%)$ & Count & Percent $(\%)$ \\
\hline Biological process & GO:0008150 & 300 & 23 & 544 & 20 \\
\hline Cellular component & GO:0005575 & 290 & 21 & 531 & 18 \\
\hline Cell & GO:0005623 & 250 & 18 & 475 & 16 \\
\hline Metabolic process & GO:0008152 & 219 & 17 & 424 & 16 \\
\hline Cellular process & GO:0009987 & 196 & 15 & 382 & 14 \\
\hline Response to stress & GO:0006950 & 87 & 7 & 200 & 7 \\
\hline Transport & GO:0006810 & 87 & 7 & 115 & 4 \\
\hline Multicellular organismal development & GO:0007275 & 54 & 4 & 126 & 5 \\
\hline Response to endogenous stimulus & GO:0009719 & 49 & 4 & 107 & 4 \\
\hline Cellular component organization & GO:0016043 & 48 & 4 & 84 & 3 \\
\hline Response to external stimulus & GO:0009605 & 48 & 4 & 106 & 4 \\
\hline Response to abiotic stimulus & GO:0009628 & 42 & 3 & 141 & 5 \\
\hline Response to biotic stimulus & GO:0009607 & 35 & 3 & 78 & 3 \\
\hline Post-embryonic development & GO:0009791 & 34 & 3 & 86 & 3 \\
\hline Reproduction & GO:0000003 & 30 & 2 & 88 & 3 \\
\hline Anatomical structure morphogenesis & GO:0009653 & 27 & 2 & 53 & 2 \\
\hline Sequence-specific DNA binding transcription factor activity & GO:0003700 & 23 & 2 & 53 & 2 \\
\hline Death & GO:0016265 & 13 & 1 & 11 & 0 \\
\hline Growth & GO:0040007 & 12 & 1 & 22 & 1 \\
\hline Regulation of gene expression, epigenetic & GO:0040029 & 11 & 1 & 4 & 0 \\
\hline Embryo development & GO:0009790 & 7 & 1 & 35 & 1 \\
\hline Pollination & GO:0009856 & 3 & 0 & 8 & 0 \\
\hline Abscission & GO:0009838 & 2 & 0 & 7 & 0 \\
\hline Translation regulator activity & GO:0045182 & 0 & 0 & 1 & 0 \\
\hline
\end{tabular}

The molecular function was associated with genes in response to $5 \%$ glucose treatment including catalytic activity, hydrolase activity, transferase activity, oxidoreductase activity, binding, and transporter activity (Table 3).

Table 3. Gene ontology classification into molecular function of all genes significantly $(\mathrm{P}<0.05)$ differentially expressed in response to $5 \%$ glucose.

\begin{tabular}{|c|c|c|c|c|c|}
\hline \multirow[t]{2}{*}{ Description } & \multirow[t]{2}{*}{ GO } & \multicolumn{2}{|c|}{ Downregulated } & \multicolumn{2}{|c|}{ Upregulated } \\
\hline & & Count & Percent (\%) & Count & Percent (\%) \\
\hline Catalytic activity & GO:0003824 & 110 & 26 & 234 & 32 \\
\hline Hydrolase activity & GO:0016787 & 41 & 10 & 76 & 10 \\
\hline Transferase activity & GO:0016740 & 32 & 8 & 67 & 9 \\
\hline Oxidoreductase activity & GO:0016491 & 27 & 6 & 66 & 9 \\
\hline Protein binding & GO:0005515 & 24 & 6 & 22 & 3 \\
\hline Cation binding & GO:0043169 & 23 & 5 & 28 & 4 \\
\hline Metal ion binding & GO:0046872 & 21 & 5 & 28 & 4 \\
\hline Transporter activity & GO:0005215 & 20 & 5 & 30 & 4 \\
\hline Nucleotide binding & GO:0000166 & 17 & 4 & 15 & 2 \\
\hline Nucleic acid binding & GO:0003676 & 16 & 4 & 29 & 4 \\
\hline Electron carrier activity & GO:0009055 & 15 & 4 & 12 & 2 \\
\hline Transcription factor activity & GO:0003700 & 14 & 3 & 28 & 4 \\
\hline DNA binding & GO:0003677 & 14 & 3 & 29 & 4 \\
\hline Transcription factor activity & GO:0003700 & 14 & 3 & 28 & 4 \\
\hline Lipid binding & GO:0008289 & 11 & 3 & 13 & 2 \\
\hline Peroxidase activity & GO:0004601 & 7 & 2 & 9 & 1 \\
\hline Enzyme regulator activity & GO:0030234 & 7 & 2 & 8 & 1 \\
\hline Signal transducer activity & GO:0004871 & 5 & 1 & 0 & 0 \\
\hline Carbohydrate binding & GO:0030246 & 5 & 1 & 5 & 1 \\
\hline Nutrient reservoir activity & GO:0045735 & 0 & 0 & 5 & 1 \\
\hline
\end{tabular}


Further analysis of DEGs with transferase activity showed that approximately $31 \%$ were related to the transfer of aglycosyl group, $26 \%$ to the transfer of phosphorus-containing groups, $20 \%$ to the transfer of acyl groups, $9 \%$ to the transfer of 1 -carbon groups, $9 \%$ to the transfer of alkyl or aryl (other than methyl) groups, and 5\% to the transfer of nitrogenous groups, as shown in Figure 2b. Additionally, detailed analysis of DEGs with hydrolase activity showed that approximately $44 \%$ were related to hydrolase activity towards ester bonds, $30 \%$ towards glycosyl bonds, $14 \%$ towards peptidase activity, $7 \%$ towards acid anhydrides, and $5 \%$ towards serine hydrolase activity (Figure 2c).

The cellular component is a prediction of gene localization. GO terms involved in the intracellular region, cytoplasm, membrane, and extracellular region were enriched (Table 4).

Table 4. Gene ontology classification into cellular component of all genes significantly $(\mathrm{P}<0.05)$ differentially expressed in response to $5 \%$ glucose.

\begin{tabular}{|c|c|c|c|c|c|}
\hline \multirow[t]{2}{*}{ Description } & \multirow[t]{2}{*}{ GO } & \multicolumn{2}{|c|}{ Downregulated } & \multicolumn{2}{|c|}{ Upregulated } \\
\hline & & Count & Percent $(\%)$ & Count & Percent (\%) \\
\hline Molecular function & GO:0003674 & 301 & 62 & 536 & 65 \\
\hline Binding & GO:0005488 & 176 & 36 & 275 & 33 \\
\hline Intracellular & GO:0005622 & 216 & 15 & 432 & 15 \\
\hline Cytoplasm & GO:0005737 & 141 & 10 & 344 & 12 \\
\hline Nucleus & GO:0005634 & 118 & 8 & 207 & 7 \\
\hline Membrane & GO:0016020 & 103 & 7 & 199 & 7 \\
\hline Extracellular region & GO:0005576 & 89 & 6 & 165 & 6 \\
\hline Plastid & GO:0009536 & 48 & 3 & 143 & 5 \\
\hline Mitochondrion & GO:0005739 & 34 & 2 & 96 & 3 \\
\hline Vacuole & GO:0005773 & 33 & 2 & 86 & 3 \\
\hline Cytosol & GO:0005829 & 21 & 1 & 64 & 2 \\
\hline External encapsulating structure & GO:0030312 & 16 & 1 & 35 & 1 \\
\hline Golgi apparatus & GO:0005794 & 16 & 1 & 19 & 1 \\
\hline Ribosome & GO:0005840 & 8 & 1 & 12 & 0 \\
\hline Thylakoid & GO:0009579 & 7 & 0 & 18 & 1 \\
\hline Endoplasmic reticulum & GO:0005783 & 6 & 0 & 22 & 1 \\
\hline Endosome & GO:0005768 & 3 & 0 & 4 & 0 \\
\hline Extracellular space & GO:0005615 & 2 & 0 & 1 & 0 \\
\hline Nuclear envelope & GO:0005635 & 1 & 0 & 1 & 0 \\
\hline Nucleolus & GO:0005730 & 1 & 0 & 9 & 0 \\
\hline Peroxisome & GO:0005777 & 1 & 0 & 16 & 1 \\
\hline Cytoskeleton & GO:0005856 & 0 & 0 & 1 & 0 \\
\hline Nucleoplasm & GO:0005654 & 0 & 0 & 5 & 0 \\
\hline
\end{tabular}

\section{High concentrations of glucose altered numerous genes involved in plant carbohy-} drate metabolism

Because a large group of genes regulated by high concentrations of glucose were found to be involved in metabolic processes, we further categorized these genes according to their roles in plant carbohydrate metabolism. A schematic of plant carbon metabolism affected by glucose is shown in Figure 3. The green and red lines indicate metabolic processes that were upregulated and downregulated by $5 \%$ glucose. Carbon metabolism-related genes showing at least 2-fold changes by glucose treatment were identified (Table 5). The phosphofructokinase and phosphoenolpyruvate carboxykinase of the 2 key enzyme involved in glycolysis were markedly upregulated by $5 \%$ glucose. Moreover, isocitrate lyase, malate 
synthase, beta-glucosidase 28 , glucose-1-phosphate adenylyltransferase family protein encoding for the large subunit of ADP-glucose pyrophosphorylase, which catalyzes the first rate-limiting step in starch biosynthesis, were increased by 10 - to 100 -fold. Moreover, cell wall metabolism-associated genes were also affected by glucose treatment, such as the cell wall invertase, pectate lyase family protein, chitinase, and plant invertase/pectin methylesterase inhibitor superfamily.

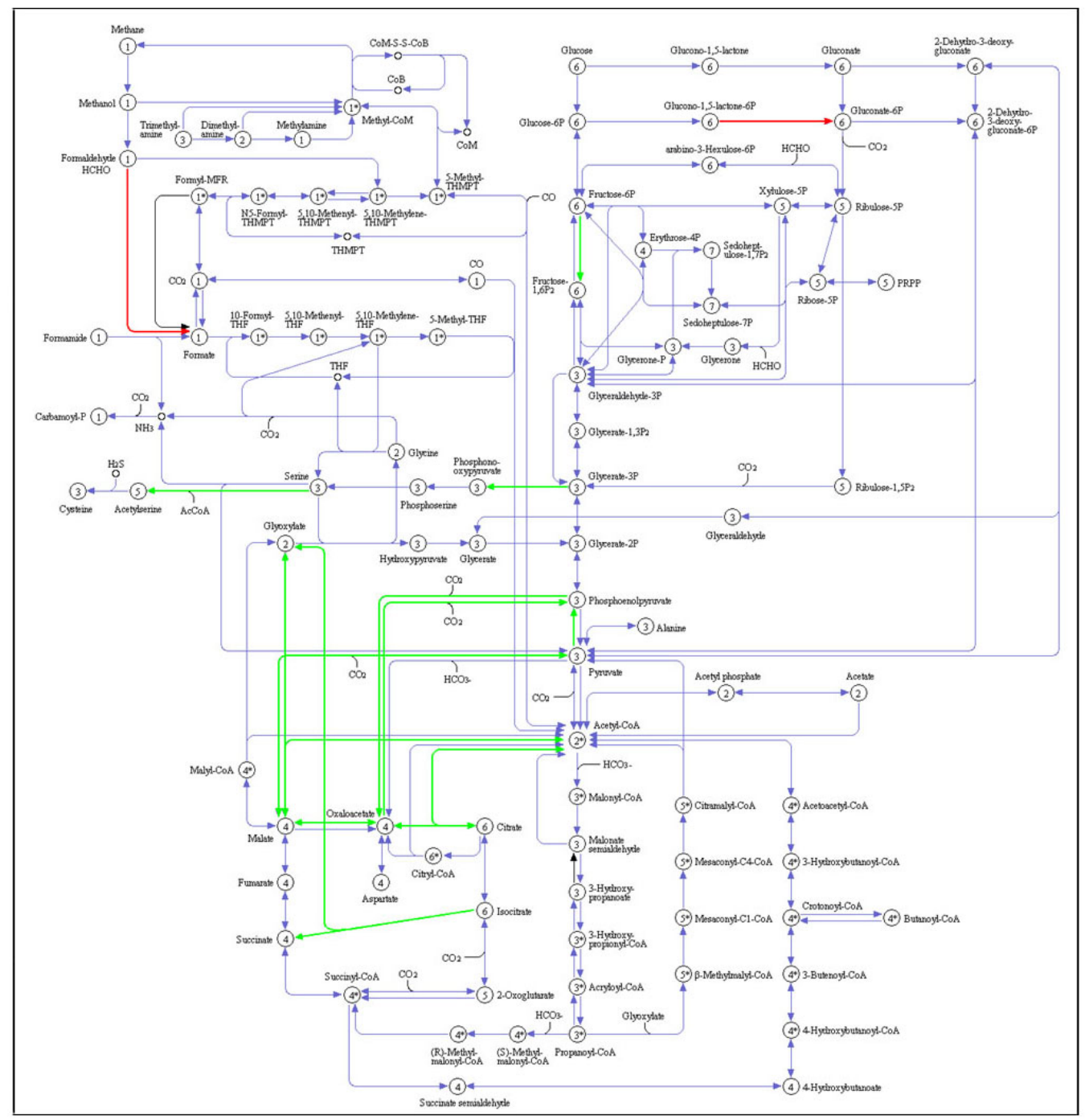

Figure 3. Schematic of plant carbon metabolism affected by glucose. The green and red lines indicate metabolic processes that were upregulated and downregulated by $5 \%$ glucose, respectively. The metabolism schema was prepared using the KEGG web service (www.genome.jp/kegg/). 
Table 5. Genes involved in carbohydrate metabolism with expression levels affected by $5 \%$ glucose by at least 2-fold.

\begin{tabular}{|c|c|c|c|}
\hline Identifier & Fold-change & $P$ value & Annotation \\
\hline AT3G21720 & 326.3 & 3.E-11 & Isocitrate lyase \\
\hline AT5G03860 & 77.7 & 5.E-08 & Malate synthase \\
\hline AT2G44460 & 55.0 & 5.E-06 & Beta-glucosidase 28 \\
\hline AT4G39210 & 37.0 & 2.E-06 & Glucose-1-phosphate adenylyltransferase family protein \\
\hline AT3G13784 & 29.4 & 1.E-04 & Cell wall invertase 5 \\
\hline AT4G09020 & 12.6 & 4.E-04 & Isoamylase 3 \\
\hline AT1G77120 & 11.4 & 7.E-04 & Alcohol dehydrogenase 1 \\
\hline AT1G48470 & 11.3 & 3.E-03 & Glutamine synthetase $1 ; 5$ \\
\hline AT2G19900 & 10.6 & 1.E-03 & NADP-malic enzyme 1 \\
\hline AT1G45191 & 8.7 & 2.E-03 & Glycosyl hydrolase superfamily protein \\
\hline AT4G33030 & 7.7 & 3.E-03 & Sulfoquinovosyl diacylglycerol 1 \\
\hline AT4G37870 & 7.3 & 4.E-03 & Phosphoenolpyruvate carboxykinase 1 \\
\hline AT1G67750 & 6.8 & 5.E-03 & Pectate lyase family protein \\
\hline AT4G26270 & 6.6 & 9.E-03 & Phosphofructokinase 3 \\
\hline AT4G15530 & 6.4 & 6.E-03 & Pyruvate orthophosphate dikinase \\
\hline AT2G21590 & 6.2 & 8.E-03 & Glucose-1-phosphate adenylyltransferase family protein \\
\hline AT4G17770 & 6.0 & 8.E-03 & Trehalose phosphatase/synthase 5 \\
\hline AT1G02850 & 6.0 & 8.E-03 & Beta glucosidase 11 \\
\hline AT5G08380 & 6.0 & 1.E-02 & Alpha-galactosidase 1 \\
\hline AT2G42790 & 5.9 & 9.E-03 & Citrate synthase 3 \\
\hline AT1G11840 & 5.6 & 1.E-02 & Glyoxalase I homolog \\
\hline AT4G02280 & 4.7 & 2.E-02 & Sucrose synthase 3 \\
\hline AT1G14520 & 4.7 & 3.E-02 & Myo-inositol oxygenase 1 \\
\hline AT3G60140 & 4.7 & 4.E-02 & Glycosyl hydrolase superfamily protein \\
\hline AT2G22780 & 4.5 & 2.E-02 & Peroxisomal NAD-malate dehydrogenase 1 \\
\hline AT2G43100 & 4.2 & 3.E-02 & Isopropylmalate isomerase 2 \\
\hline AT3G14940 & 3.9 & 4.E-02 & Phosphoenolpyruvate carboxylase 3 \\
\hline AT3G12500 & 3.7 & 5.E-02 & Basic chitinase \\
\hline AT5G42250 & 0.3 & 4.E-02 & Zinc-binding alcohol dehydrogenase family protein \\
\hline AT5G10170 & 0.2 & 3.E-02 & Myo-inositol-1-phosphate synthase 3 \\
\hline AT5G24420 & 0.2 & 2.E-02 & 6-phosphogluconolactonase 5 \\
\hline AT1G66270 & 0.2 & 1.E-02 & Glycosyl hydrolase superfamily protein \\
\hline AT2G47550 & 0.2 & 4.E-02 & Plant invertase/pectin methylesterase inhibitor superfamily \\
\hline AT5G04970 & 0.1 & 1.E-02 & Plant invertase/pectin methylesterase inhibitor superfamily \\
\hline AT3G24230 & 0.1 & 3.E-02 & Pectate lyase family protein \\
\hline AT3G09940 & 0.1 & 6.E-03 & Monodehydroascorbate reductase \\
\hline AT5G24410 & 0.1 & 3.E-02 & 6-phosphogluconolactonase 4 \\
\hline AT4G39770 & 0.1 & 1.E-03 & Haloacid dehalogenase-like hydrolase (HAD) superfamily protein \\
\hline AT5G20250 & 0.1 & 3.E-04 & Raffinose synthase family protein \\
\hline
\end{tabular}

\section{Hormone signals with changes in response to $5 \%$ glucose}

Glucose not only acts as fuel in metabolism, but also functioned as a hormone-like signaling molecule during plant growth and development (Arenas-Huertero et al., 2000; Rolland et al., 2002; Moore et al., 2003). Here, we identified 15 hormone-related genes showing $\geq 2$-fold change in transcript levels in response to glucose (Table 6). Most DEGs were auxinrelated genes, and the auxin-responsive GH3 family protein and Pin-like 7 encoding for the auxin efflux carrier protein were upregulated; in contrast, IAA6, IAA19, IAA29, and small auxin-up RNA-like auxin-responsive protein were downregulated significantly. Three members of the pyrabactin resistance (PYR)/PYR-like (PYL) family proteins were all downregulated after 5\% glucose treatment. Moreover, protein phosphatase 2C (PP2Cs) were also affected by high concentrations of glucose. 
Table 6. Hormone signals with changes in gene expression levels of at least 2 -fold following $5 \%$ glucose treatment.

\begin{tabular}{|c|c|c|c|}
\hline Identifier & Fold-change & $P$ value & Annotation \\
\hline AT1G48660 & 45.7 & 2.E-06 & Auxin-responsive GH3 family protein \\
\hline AT2G40220 & 21.7 & 6.E-03 & Encodes a member of the DREB subfamily A-3 of ERF/AP2 transcription factor family (ABI4) \\
\hline AT1G48670 & 6.2 & 1.E-02 & Auxin-responsive GH3 family protein \\
\hline AT5G65980 & 5.0 & 2.E-02 & Auxin efflux carrier family protein \\
\hline AT1G66350 & 4.2 & 3.E-02 & Negative regulator of GA responses, member of GRAS family of transcription factors \\
\hline AT5G59220 & 4.2 & 3.E-02 & Encodes a member of the PP2C family (Clade A protein phosphatases type 2C) \\
\hline AT5G15230 & 4.0 & 4.E-02 & Encodes gibberellin-regulated protein GASA4. Promotes GA responses and exhibits redox activity \\
\hline AT3G15540 & 0.2 & 4.E-02 & Indole-3-acetic acid inducible 19 \\
\hline AT2G38310 & 0.2 & 2.E-02 & $\begin{array}{l}\text { Encodes a member of the PYR (pyrabactin resistance)/PYL(PYR1-like)/RCAR (regulatory } \\
\text { components of ABA receptor) family proteins with } 14 \text { members }\end{array}$ \\
\hline AT5G05440 & 0.2 & 1.E-02 & $\begin{array}{l}\text { Encodes a member of the PYR (pyrabactin resistance)/PYL(PYR1-like)/RCAR (regulatory } \\
\text { components of ABA receptor) family proteins with } 14 \text { members }\end{array}$ \\
\hline AT4G32280 & 0.2 & 1.E-02 & Indole-3-acetic acid inducible 29 \\
\hline AT1G22690 & 0.2 & 6.E-03 & Gibberellin-regulated family protein \\
\hline AT1G56150 & 0.1 & 1.E-02 & SAUR-like auxin-responsive protein family \\
\hline AT4G32950 & 0.1 & 1.E-02 & Protein phosphatase $2 \mathrm{C}$ family protein \\
\hline AT2G40330 & 0.1 & 9.E-04 & $\begin{array}{l}\text { Encodes a member of the PYR (pyrabactin resistance)/PYL(PYR1-like)/RCAR (regulatory } \\
\text { components of ABA receptor) family proteins with } 14 \text { members }\end{array}$ \\
\hline AT1G52830 & 0.1 & 5.E-03 & Indole-3-acetic acid 6 \\
\hline
\end{tabular}

\section{Glucose altered kinases and calcium signaling genes}

Glucose acts as a signaling molecule to influence plant growth and development. In order to understand the role of glucose in plant signaling, we identified kinases and genes involved in calcium signaling showing at least 2-fold changes in RNA-seq data. As shown in Table 7, all but 6 kinases identified showing a change in transcript abundance of at least 2 -fold in response to $5 \%$ glucose treatment were downregulated, including CBL-interacting protein kinase and mitogen-activated protein kinase. However, metabolic-related kinases such as phosphoenolpyruvate carboxykinase, phosphofructokinase, pyruvate orthophosphate dikinase, and APS-kinase encoding adenosine-5'-phosphosulfate-kinase were upregulated. Calcium signals are also key regulators of the plant response to environmental stimuli. Interestingly, only 3 genes identified involved in calcium signaling according to our RNA-seq data were regulated in response to glucose.

\section{Transcription factors affected by glucose}

A total of 36 transcription factors showed at least 2-fold changes in response to high concentrations of glucose (Table 8). Interestingly, all but 7 transcription factors were upregulated, including 5 basic helix-loop-helix DNA-binding superfamily proteins, 4 MYB domain proteins, 2 NAC domain-containing proteins, and 2 nuclear factors.

\section{Transporter-associated genes were influenced by glucose treatment}

Our RNA-seq data showed that phosphate transporters, sulfate transporters, and multidrug and multidrug and toxic efflux transporters were all upregulated by $5 \%$ glucose treatment. Additionally, the transcriptional levels of some $\mathrm{K}^{+}$and nitrate transporters were altered 
under high concentrations of glucose. The high affinity $\mathrm{K}^{+}$transporter (HAK5) was markedly upregulated after glucose treatment, whereas another $\mathrm{K}^{+}$transporter, SKOR encoding for stellar $\mathrm{K}^{+}$outward rectifier were significantly downregulated, as shown in Table 9. Moreover, the 2 nitrate transporters showed a different expression pattern compared to NRT1.7 was reduced, but NRT2.3 was increased after exposure to a high concentration of glucose, indicating nitrate transporter sense glucose signals in different pathways. Interestingly, the transcript levels of some cation transporters were also affected by glucose treatment, with the cation efflux family protein $(M T P 8)$ and cation exchangers $(C A X 3$ and $C A X 7)$ upregulated and the cation $/ \mathrm{H}^{+}$ exchanger (CHX20) downregulated. Amino acid transporter (AT5G04770) as well as sugar transporter $S T P 1$ encoding a $\mathrm{H}^{+}$/hexose cotransporter were significantly reduced by glucose.

Table 7. Kinases and calcium signals showing changes in gene expression levels of at least 2-fold following $5 \%$ glucose treatment.

\begin{tabular}{|c|c|c|c|}
\hline Identifier & Fold-change & $P$ value & Annotation \\
\hline \multicolumn{4}{|l|}{ Kinases } \\
\hline AT5G11410 & 7.3 & 4.E-02 & Protein kinase superfamily protein \\
\hline AT4G37870 & 7.3 & 4.E-03 & Phosphoenolpyruvate carboxykinase 1 \\
\hline AT4G26270 & 6.6 & 9.E-03 & Phosphofructokinase 3 \\
\hline AT4G15530 & 6.4 & 6.E-03 & Pyruvate orthophosphate dikinase \\
\hline AT4G39940 & 5.0 & 2.E-02 & APS-kinase 2 \\
\hline AT4G23250 & 4.0 & 4.E-02 & Kinases; protein kinases \\
\hline AT4G38470 & 0.3 & 5.E-02 & ACT-like protein tyrosine kinase family protein \\
\hline AT4G14480 & 0.2 & 4.E-02 & Protein kinase superfamily protein \\
\hline AT3G46280 & 0.2 & 2.E-02 & Protein kinase-related \\
\hline AT5G39020 & 0.2 & 4.E-02 & Malectin/receptor-like protein kinase family protein \\
\hline AT5G45820 & 0.2 & 2.E-02 & CBL-interacting protein kinase 20 \\
\hline AT1G65190 & 0.2 & 2.E-02 & Protein kinase superfamily protein \\
\hline AT1G11340 & 0.2 & 3.E-02 & S-locus lectin protein kinase family protein \\
\hline AT4G11460 & 0.2 & 1.E-02 & Cysteine-rich RLK (RECEPTOR-like protein kinase) 30 \\
\hline AT5G59680 & 0.2 & 2.E-02 & Leucine-rich repeat protein kinase family protein \\
\hline AT3G22060 & 0.1 & 4.E-03 & Receptor-like protein kinase-related family protein \\
\hline AT5G24100 & 0.1 & 9.E-03 & Leucine-rich repeat protein kinase family protein \\
\hline AT2G19190 & 0.1 & 1.E-02 & FLG22-induced receptor-like kinase 1 \\
\hline AT4G23200 & 0.1 & 3.E-03 & Cysteine-rich RLK (RECEPTOR-like protein kinase) 12 \\
\hline AT1G29720 & 0.1 & 2.E-03 & Leucine-rich repeat transmembrane protein kinase \\
\hline AT2G28960 & 0.1 & 4.E-03 & Leucine-rich repeat protein kinase family protein \\
\hline AT4G36450 & 0.1 & 4.E-02 & Mitogen-activated protein kinase 14 \\
\hline AT4G00970 & 0.1 & 2.E-03 & Cysteine-rich RLK (RECEPTOR-like protein kinase) 41 \\
\hline \multicolumn{4}{|c|}{ Genes involved in calcium signaling } \\
\hline AT1G23140 & 6.6 & 1.E-02 & Calcium-dependent lipid-binding (calb domain) family protein \\
\hline AT1G29025 & 0.2 & 4.E-02 & Calcium-binding EF-hand family protein \\
\hline AT3G59370 & 0.1 & 8.E-03 & Vacuolar calcium-binding protein-related \\
\hline
\end{tabular}

\section{Validation of RNA sequencing data by quantitative real-time-PCR}

We conducted real-time PCR analysis to confirm the validity of the changes observed between treatments. A total of 10 selected genes showing transcript changes in response to glucose treatment in the RNA-seq analysis were examined using real-time-PCR. Expression levels were detected in these RNA samples. For all 10 genes, transcript levels determined by real-time PCR analysis were similar to those detected using RNA-seq (Figure 4), indicating the reliability of the RNA-seq data. 
Table 8. Transcription factors with changes in gene expression levels of at least 2 -fold following $5 \%$ glucose treatment.

\begin{tabular}{|c|c|c|c|}
\hline Identifier & Fold-change & $P$ value & Annotation \\
\hline AT3G50330 & 67.7 & 8.E-06 & Basic helix-loop-helix (bhlh) DNA-binding superfamily protein \\
\hline AT1G56650 & 54.0 & 2.E-06 & Production of anthocyanin pigment 1 \\
\hline AT1G72570 & 51.9 & 3.E-05 & Integrase-type DNA-binding superfamily protein \\
\hline AT3G14880 & 23.2 & 1.E-03 & BEST Arabidopsis thaliana protein match is: transcription factor-related \\
\hline AT1G18710 & 18.2 & 1.E-04 & Myb domain protein 47 \\
\hline AT5G49330 & 13.9 & 9.E-04 & Myb domain protein 111 \\
\hline AT4G09820 & 13.6 & 8.E-04 & Basic helix-loop-helix (bhlh) DNA-binding superfamily protein \\
\hline AT5G65320 & 11.1 & 6.E-03 & Basic helix-loop-helix (bhlh) DNA-binding superfamily protein \\
\hline AT3G62610 & 9.6 & 1.E-02 & Myb domain protein 11 \\
\hline AT1G03790 & 9.2 & 2.E-03 & Zinc finger $\mathrm{C}-\mathrm{x} 8-\mathrm{C}-\mathrm{x} 5-\mathrm{C}-\mathrm{x} 3-\mathrm{H}$ type family protein \\
\hline AT2G43000 & 9.2 & 3.E-03 & NAC domain containing protein 42 \\
\hline AT2G17040 & 8.3 & 8.E-03 & NAC domain containing protein 36 \\
\hline AT2G27300 & 7.9 & 2.E-02 & NTM1-like 8 \\
\hline AT5G06250 & 7.2 & 9.E-03 & AP2/B3-like transcriptional factor family protein \\
\hline AT5G43840 & 6.8 & 9.E-03 & Heat shock transcription factor A6A \\
\hline AT4G30180 & 6.2 & 3.E-02 & Sequence-specific DNA binding transcription factors \\
\hline AT3G61950 & 6.1 & 1.E-02 & Basic helix-loop-helix (bhlh) DNA-binding superfamily protein \\
\hline AT2G35700 & 5.9 & 3.E-02 & ERF family protein 38 \\
\hline AT5G57390 & 5.7 & 1.E-02 & AINTEGUMENTA-like 5 \\
\hline AT4G18650 & 5.6 & 3.E-02 & Transcription factor-related \\
\hline AT2G37260 & 5.4 & 2.E-02 & WRKY family transcription factor family protein \\
\hline AT5G67060 & 5.4 & 1.E-02 & Basic helix-loop-helix (bhlh) DNA-binding superfamily protein \\
\hline AT5G06510 & 5.4 & 2.E-02 & Nuclear factor Y, subunit A10 \\
\hline AT5G07690 & 5.0 & 2.E-02 & Myb domain protein 29 \\
\hline AT1G54160 & 5.0 & 3.E-02 & Nuclear factor Y, subunit A5 \\
\hline AT1G68880 & 4.9 & 3.E-02 & Basic leucine-zipper 8 \\
\hline AT2G46680 & 4.3 & 3.E-02 & Homeobox 7 \\
\hline AT1G66350 & 4.2 & 3.E-02 & RGA-like 1 \\
\hline AT3G51910 & 0.2 & 4.E-02 & Heat shock transcription factor $\mathrm{A} 7 \mathrm{~A}$ \\
\hline AT1G36060 & 0.2 & 2.E-02 & Integrase-type DNA-binding superfamily protein \\
\hline AT2G21650 & 0.1 & 4.E-02 & Homeodomain-like superfamily protein \\
\hline AT1G24260 & 0.1 & 7.E-03 & K-box region and MADS-box transcription factor family protein \\
\hline AT1G71030 & 0.1 & 1.E-03 & MYB-like 2 \\
\hline AT3G60490 & 0.1 & 2.E-02 & Integrase-type DNA-binding superfamily protein \\
\hline AT5G28770 & 0.1 & 7.E-04 & Bzip transcription factor family protein \\
\hline
\end{tabular}

Table 9. Transporters affected by glucose treatment.

\begin{tabular}{|c|c|c|c|c|}
\hline Groups & Identifier & Fold-change & $P$ value & Annotation \\
\hline \multirow[t]{2}{*}{$\mathrm{K}^{+}$transporter } & AT4G13420 & 8.4 & 1.E-02 & High-affinity $\mathrm{K}^{+}$transporter 5 \\
\hline & AT3G02850 & 0.1 & 7.E-03 & STELAR $\mathrm{K}^{+}$outward rectifier \\
\hline \multirow[t]{2}{*}{ Nitrate transporter } & AT1G69870 & 8.5 & 2.E-03 & Nitrate transporter 1.7 \\
\hline & AT5G60780 & 0.1 & 1.E-02 & Nitrate transporter 2.3 \\
\hline \multirow[t]{2}{*}{ Phosphate transporter } & AT2G32830 & 17.6 & 6.E-04 & Phosphate transporter 1.5 \\
\hline & AT3G48850 & 7.2 & 1.E-02 & Phosphate transporter 3.2 \\
\hline \multirow[t]{4}{*}{ Cation transporter } & AT3G58060 & 25.1 & 3.E-04 & Cation efflux family protein \\
\hline & AT3G51860 & 4.9 & 2.E-02 & Cation exchanger 3 \\
\hline & AT5G17860 & 3.9 & 4.E-02 & Calcium exchanger 7 \\
\hline & AT3G53720 & 0.3 & 5.E-02 & Cation/ $\mathrm{H}^{+}$exchanger 20 \\
\hline \multirow[t]{2}{*}{ Sulfate transporter } & AT4G02700 & 8.8 & 2.E-03 & Sulfate transporter 3.2 \\
\hline & AT3G12520 & 4.2 & 3.E-02 & Sulfate transporter 4.2 \\
\hline Channel & AT1G01340 & 0.2 & 2.E-02 & Cyclic nucleotide gated channel 10 \\
\hline \multirow[t]{5}{*}{ MATE efflux family protein } & AT2G34360 & 8.8 & 3.E-02 & MATE efflux family protein \\
\hline & AT2G04050 & 8.2 & 9.E-03 & MATE efflux family protein \\
\hline & AT3G03620 & 7.4 & 4.E-02 & MATE efflux family protein \\
\hline & AT1G71140 & 4.5 & 3.E-02 & MATE efflux family protein \\
\hline & AT4G21910 & 3.7 & 5.E-02 & MATE efflux family protein \\
\hline Amino acid transporter & AT5G04770 & 0.2 & 2.E-02 & Cationic amino acid transporter 6 \\
\hline \multirow[t]{2}{*}{ Sugar transporter } & AT4G36670 & 0.2 & 7.E-03 & $\begin{array}{l}\text { Carbohydrate transmembrane transporter activity, } \\
\text { sugar: hydrogen symporter activity }\end{array}$ \\
\hline & AT1G11260 & 0.1 & 5.E-03 & Encodes a $\mathrm{H}^{+} /$hexose cotransporter \\
\hline
\end{tabular}



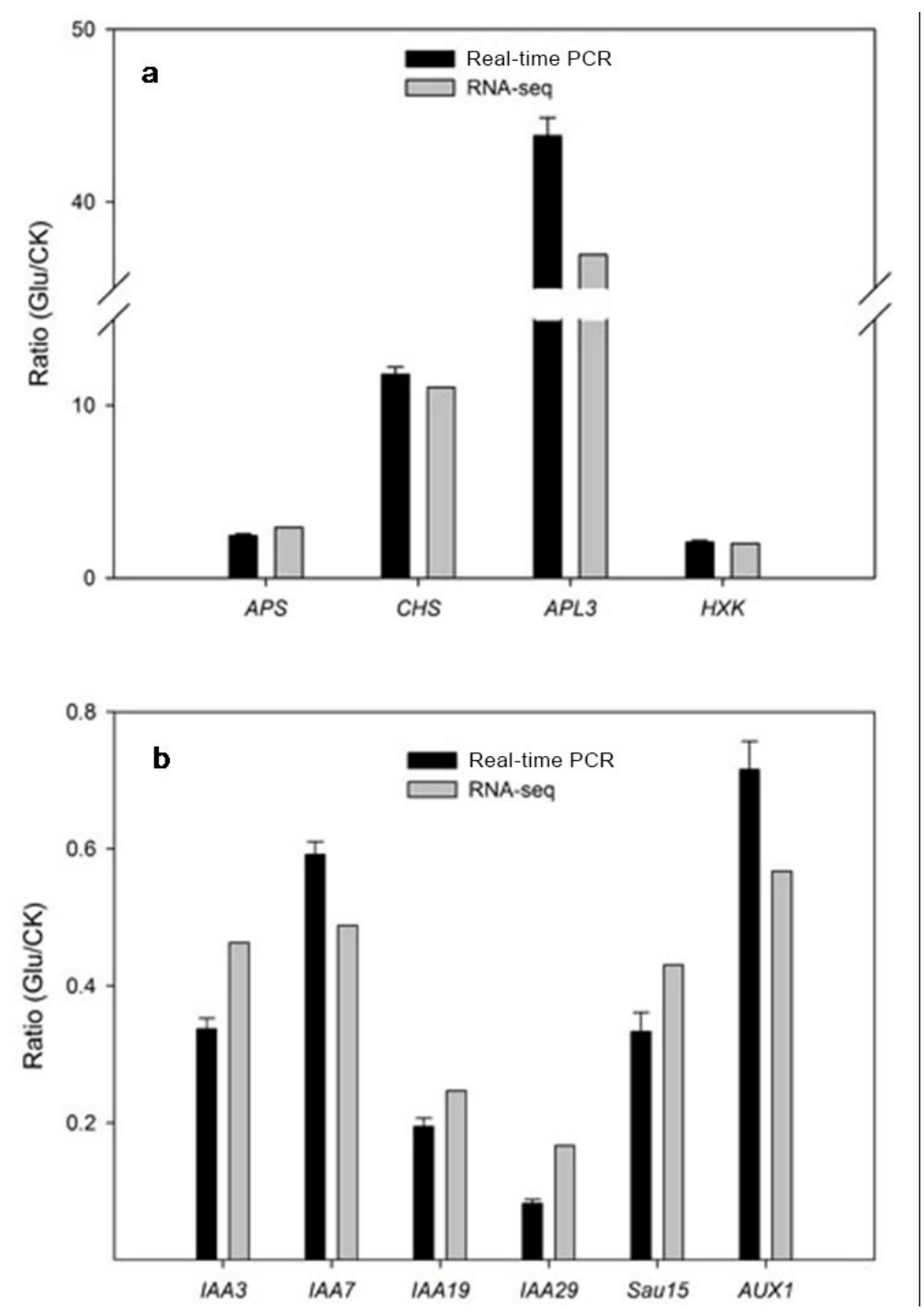

Figure 4. Validation of RNA sequencing data of genes selected by qRT-PCR. Glu/CK ratios in RNA-seq data of 10 tested genes compared with qRT-PCR results. Sequences of primers used for PCR are listed in Table S1.

\section{DISCUSSION}

Plants are capable of monitoring the nutrients available and adapting their transcriptional responses and metabolic processes to this information. Nutrients function not only as food but also as signals for growth and development. For example, sugars, provide skeletons for organic molecules and store energy for biochemical reactions. Sugars also act as signaling molecules involved in both cellular metabolic processes and abiotic and biotic stress response (Gibson, 2005; Rolland et al., 2006). Sugars were reported to influence plant seeds germination, hypocotyls elongation, cotyledon expansion, root development, true leaf formation, 
flowering, and senescence (Rolland et al., 2006). High concentrations of exogenous sugars were found to suppress hypocotyls elongation, cotyledon greening, and expansion and root development during early seedling development in Arabidopsis (Rolland et al., 2006). However, detailed studies of the sugars associated with early seedling development are lacking; there are few reports regarding the whole genome scale analysis of the effects of sugars on Arabidopsis. In this study, we examined transcriptional regulation involved in metabolism processes and signaling action in Arabidopsis in response to high concentrations of glucose.

RNA-seq data showed that the transcript levels of 863 genes were altered by glucose treatment, and a large number of DEGs were related to other nutrient metabolic processes such as nitrogen (Coruzzi and Bush, 2001; Coruzzi and Zhou, 2001). Sugar metabolic processes are involved in regulating photosynthetic activity at the transcriptional level, and Sheen (1990) reported that 7 photosynthetic genes were downregulated by glucose and sucrose in maize mesophyll protoplasts.

Plant glucose signaling was reported to have a complicated interaction with hormone signaling networks. Auxin is a very important hormone for plant growth as well as for development. Both auxin and sugar are very important for plants growth and the regulation of similar processes (Mishra et al., 2009). gin2, a glucose insensitive mutant, was reported to be resistant to exogenous auxin application (Moore et al., 2003). In hlsl, a loss of function mutation in the HOOKLESS1 gene, exogenous IAA application repressed sugar-induced gene expression and activated some auxin response genes such as the AUR3 encoding GH3-like protein (Ohto et al., 2006). AUR3 GH3-like protein conjugates free IAA to amino acids; the upregulation of GH3-like genes can lead to a decrease in the free IAA level in plants (Staswick et al., 2005). According to our RNA-seq data, the transcript abundance of the 2 putative genes encoding for auxin-responsive GH3 family proteins were markedly increased, indicating that the free IAA level in seedlings under 5\% glucose treatment may be decreased and subsequently show inhibited growth. Additionally, the RNA-seq data showed that the expression of the IAA6, $I A A 19$, and $I A A 29$ genes was reduced, indicating alterations in auxin biosynthesis in seedlings under $5 \%$ glucose treatment. Sugars also affected gibberellin metabolism/transport. For example, gibberellin levels in barley (Perata et al., 1997) and rice (Yu et al., 1996) embryos were decreased following the application of glucose. The expression levels in seedlings treated with $5 \%$ glucose showed alterations in the $R G A$-like 1 encoding negative regulator of GA response, GASA4 encoding gibberellins-regulated protein GASA4, and putative gibberellins-regulated family gene (AT1G22690) (Table 6), indicating that these genes coordinately regulate gibberellin levels in glucose-treated seedlings in Arabidopsis. Glucose has been shown to affect the expression and/or activity levels of ABA pathways, and ABA levels were increased in young seedlings by exogenous application of glucose in Arabidopsis (Arenas-Huertero et al., 2000; Cheng et al., 2002). Moreover, the transcript levels of $A B I 3, A B I 4$, and $A B I 5$ were induced in Arabidopsis seedlings treated with glucose (Arenas-Huertero et al., 2000; Cheng et al., 2002). According to our RNA-seq data, high levels of glucose significantly induced the expression of ABI4 in Arabidopsis; $A B I 3$ and $A B I 5$ were also upregulated by approximately3-fold (data not shown). The ABA signaling gene $A B I 4$ codes for a member of the DREB subfamily A-3 of ERF/AP2 transcription factor family (Arenas-Huertero et al., 2000) and was also associated with the activation of glucose-1-phosphate adenylyltransferase family protein by glucose and ABA (Arenas-Huertero et al., 2000). Glucose-1-phosphate adenylyltransferase family protein encodes a large subunit of ADP-glucose pyrophosphorylase and was remarkably enhanced by exogenous application of high levels of glucose (Table 2). PP2Cs are important phospha- 
tases that played vital roles in abscisic acid (ABA) signaling. PYR1 (pyrabactin Pyrabactin resistance 1), a member of the PYR/PYL family protein, is necessary for ABA signaling. $\mathrm{ABA}$ binds to pyrabactin resistance 1, which in turn binds to and inhibits PP2Cs, suggesting that PYR/PYLs are ABA receptors acting as negative regulator that control ABA signaling by inhibiting PP2Cs (Park et al., 2009). PYR4, PYR5, and PYR6 as well as 2 PP2Cs were transcriptionally regulated, indicating that the ABA signaling network was altered by glucose treatment. AtbZIP63, which codes for a basic-leucine zipper transcription factor, was repressed by 10 -fold by glucose treatment (Table 8). Previous reports showed that the repression of bZIP63 by high levels of glucose $(6 \%)$ was regulated by ABA (Matiolli et al., 2011).

The expression of multiple transcription factors and kinase and calcium signaling genes were regulated by glucose treatment. The transcript of MYB75/PAP1, which encodes a putative MYB domain containing a transcription factor involved in anthocyanin metabolism, was induced by glucose (Table 8). MYB75/PAP1 expression was strongly dependent on HY5, which encodes a component of light-signaling pathways, suggesting that anthocyanin biosynthesis is regulated by HY5 by a bifurcate regulation mechanism (Shin et al., 2013). MYB75/PAP1 can activate other MYB members related to anthocyanin biosynthesis, including PAP2, MYB113, and MYB114 (Shin et al., 2013). The expression of MYB75/PAP1 was positively regulated by sugars (Das et al., 2012). Overexpression of MYB75/PAP1 also resulted in increased expression of anthocyanin biosynthetic genes (Borevitz et al., 2000), indicating that phenylpropanoid biosynthetic genes were induced by glucose via elevated expression of MYB transcription factors. The expression of other transcription factors, including AP2, bZIP, homeodomain, NAC, Zine finger protein, and heat-shock transcription factor, were also influenced by glucose. Tandem $\mathrm{CCCH}$ zinc finger protein 4 is related to light-dependent seed germination (Kim et al., 2008), and its expression is regulated by ABA and gibberellins (Bogamuwa and Jang, 2013), indicating that glucose-induced tandem $\mathrm{CCCH}$ zinc finger protein 4 was associated with the $\mathrm{ABA}$ or gibberellins signal network during seed germination. Calcium acted as a ubiquitous secondary messenger in plant cells. CBLs are $\mathrm{Ca}^{2+}$ sensors that modulate CIPKs in response to various abiotic stresses. Recent studies have shown that expression of CBL1 and CIPK14 was induced by glucose treatment, and knockout mutations in CIPK14 and CBL1 resulted in hypersensitivity to glucose treatment ( $\mathrm{Li}$ et al., 2013; Yan et al., 2014). Moreover, CIPK14 was reported to interact with CBL2, CBL3, and CBL9 (Yan et al., 2014). According to our RNA-seq data, the CIPK20 and 3 putative $\mathrm{Ca} 2+$ binding proteins were regulated by glucose, indicating that CBLs and CIPKs are involved in plant glucose signaling.

Acquisition of mineral ions must match the nutrient demand of whole plants. The expression of ion transport systems are regulated on the basis of nutritional status. For example, the uptake of $\mathrm{NO}_{3}^{-}$is regulated by sugars and carboxylic acids in the roots (Touraine et al., 1992; Delhon et al., 1996). AtKUP2 ( $\mathrm{K}^{+}$transporter), AtNRT1.1, AtNRT2.1 ( $\mathrm{NO}_{3}{ }^{-}$transporter), AtHST1 (a high-affinity sulfate transporter), AtPt2, and AtKup2 transcript levels were increased by sucrose supply, whereas AtSKOR was reduced (Lejay et al., 2003). This agrees with our RNA-seq data, as shown in Table 9. Twenty-one genes involved in nutrient transport activity, including $\mathrm{N}, \mathrm{P}, \mathrm{K}, \mathrm{Ca}, \mathrm{S}$, and organics were found to be regulated by glucose (Table 9). In Arabidopsis, there are 56 genes classified into the multidrug and toxic efflux transporter family, most of which are defined as putative (Li et al., 2002). Five multidrug and toxic efflux transporter family genes were upregulated by glucose, indicating they may be involved in drug resistance or detoxification. Sugar transporter protein 1 and polyol transporter 6 were repressed by high levels of glucose. In Arabidopsis, numerous AtSTPs have been extensively 
studied and found to mediate the absorption of hexoses from the apoplastic space across the plasma membrane (Buttner, 2010). In Arabidopsis, overexpression of sugar transporter protein 1 driven by the $35 \mathrm{~S}$ promoter markedly increased glucose absorption (Baier et al., 2004). The capacity to uptake D-glucose was $60 \%$ lower in mutant stp1 seedlings than in wild-type, and AtSTPs were repressed by glucose and sucrose (except AtSTP3 and AtSTP4) (Buttner, 2010).

Our transcriptional-level assays provide a basic understanding of glucose-mediated molecular mechanisms regulating early seedling development.

\section{ACKNOLWEDGMENTS}

Research supported by the National Science Foundation of China (\#91125028).

\section{Supplementary material}

\section{REFERENCES}

Arenas-Huertero F, Arroyo A, Zhou L, Sheen J, et al. (2000). Analysis of Arabidopsis glucose insensitive mutants, gin5 and gin6, reveals a central role of the plant hormone ABA in the regulation of plant vegetative development by sugar. Genes Dev. 14: 2085-2096.

Baier M, Hemmann G, Holman R, Corke F, et al. (2004). Characterization of mutants in Arabidopsis showing increased sugar-specific gene expression, growth, and developmental responses. Plant Physiol. 134: 81-91.

Bogamuwa S and Jang JC (2013). The Arabidopsis tandem CCCH zinc finger proteins AtTZF4, 5 and 6 are involved in light-, abscisic acid- and gibberellic acid-mediated regulation of seed germination. Plant Cell Environ. 36: 1507-1519.

Borevitz JO, Xia Y, Blount J, Dixon RA, et al. (2000). Activation tagging identifies a conserved MYB regulator of phenylpropanoid biosynthesis. Plant Cell 12: 2383-2394.

Buttner M (2010). The Arabidopsis sugar transporter (AtSTP) family: an update. Plant Biol. 12 (Suppl 1): 35-41.

Cheng WH, Endo A, Zhou L, Penney J, et al. (2002). A unique short-chain dehydrogenase/reductase in Arabidopsis glucose signaling and abscisic acid biosynthesis and functions. Plant Cell 14: 2723-2743.

Coruzzi G and Bush DR (2001). Nitrogen and carbon nutrient and metabolite signaling in plants. Plant Physiol. 125: 61-64.

Coruzzi GM and Zhou L (2001). Carbon and nitrogen sensing and signaling in plants: emerging 'matrix effects'. Curr. Opin. Plant Biol. 4: 247-253.

Das PK, Shin DH, Choi SB, Yoo SD, et al. (2012). Cytokinins enhance sugar-induced anthocyanin biosynthesis in Arabidopsis. Mol. Cells 34: 93-101.

Delhon P, Gojon A, Tillard P and Passama L (1996). Diurnal regulation of NO3- uptake in soybean plants4. Dependence on current photosynthesis and sugar availability to the roots. J. Exp. Bot. 47: 893-900.

Du Z, Zhou X, Ling Y, Zhang Z, et al. (2010). agriGO: a GO analysis toolkit for the agricultural community. Nucleic Acids Res. 38: W64-W70.

Gibson SI (2005). Control of plant development and gene expression by sugar signaling. Curr. Opin. Plant Biol. 8: 93-102.

Gibson SI, Laby RJ and Kim D (2001). The sugar-insensitive1 (sis1) mutant of Arabidopsis is allelic to ctr1. Biochem. Biophys. Res. Commun. 280: 196-203.

Kim DH, Yamaguchi S, Lim S, Oh E, et al. (2008). SOMNUS, a CCCH-type zinc finger protein in Arabidopsis, negatively regulates light-dependent seed germination downstream of PIL5. Plant Cell 20: 1260-1277.

Koch K (2004). Sucrose metabolism: regulatory mechanisms and pivotal roles in sugar sensing and plant development. Curr. Opin. Plant Biol. 7: 235-246.

Kudla J, Batistic O and Hashimoto K (2010). Calcium signals: the lead currency of plant information processing. Plant Cell 22: 541-563.

Lee SA, Yoon EK, Heo JO, Lee MH, et al. (2012). Analysis of Arabidopsis glucose insensitive growth mutants reveals the involvement of the plastidial copper transporter PAA1 in glucose-induced intracellular signaling. Plant Physiol. 159: 1001-1012.

Lejay L, Gansel X, Cerezo M, Tillard P, et al. (2003). Regulation of root ion transporters by photosynthesis: functional importance and relation with hexokinase. Plant Cell 15: 2218-2232.

León P and Sheen J (2003). Sugar and hormone connections. Trends Plant Sci. 8: 110-116.

Li L, He Z, Pandey GK, Tsuchiya T, et al. (2002). Functional cloning and characterization of a plant efflux carrier for 
multidrug and heavy metal detoxification. J. Biol. Chem. 277: 5360-5368.

Li ZY, Xu ZS, Chen Y, He GY, et al. (2013). A novel role for Arabidopsis CBL1 in affecting plant responses to glucose and gibberellin during germination and seedling development. PLoS One 8: e56412.

Lilley JLS, Gee CW, Sairanen I, Liung K, et al. (2012). An endogenous carbon-sensing pathway triggers increased auxin flux and hypocotyl elongation. Plant Physiol. 160: 2261-2270.

Liu J, Ishitani M, Halfter U, Kim CS, et al. (2000). The Arabidopsis thaliana SOS2 gene encodes a protein kinase that is required for salt tolerance. Proc. Natl. Acad. Sci. U. S. A. 97: 3730-3734.

Matiolli CC, Tomaz JP, Duarte GT, Prado FM, et al. (2011). The Arabidopsis bZIP gene AtbZIP63 is a sensitive integrator of transient abscisic acid and glucose signals. Plant Physiol. 157: 692-705.

Mishra BS, Singh M, Aggrawal P and Laxmi A (2009). Glucose and auxin signaling interaction in controlling Arabidopsis thaliana seedlings root growth and development. PLoS One 4: e4502.

Moore B, Zhou L, Rolland F, Hall Q, et al. (2003). Role of the Arabidopsis glucose sensor HXK1 in nutrient, light, and hormonal signaling. Science 300: 332-336.

Ohto MA, Hayashi S, Sawa S, Hashimoto-Ohta A, et al. (2006). Involvement of HLS1 in sugar and auxin signaling in Arabidopsis leaves. Plant Cell Physiol. 47: 1603-1611.

Overvoorde P, Fukaki H and Beeckman T (2010). Auxin control of root development. Cold Spring Harb. Perspect Biol. 2: a001537.

Park SY, Fung P, Nishimura N, Jensen DR, et al. (2009). Abscisic acid inhibits type 2C protein phosphatases via the PYR/ PYL family of START proteins. Science 324: 1068-1071.

Perata P, Matsukura C, Vernieri P and Yamaguchi J (1997). Sugar repression of a gibberellin-dependent signaling pathway in barley embryos. Plant Cell 9: 2197-2208.

Rolland F, Moore B and Sheen J (2002). Sugar sensing and signaling in plants. Plant Cell 14 Suppl: S185-S205.

Rolland F, Baena-Gonzalez E and Sheen J (2006). Sugar sensing and signaling in plants: conserved and novel mechanisms. Annu. Rev. Plant Biol. 57: 675-709.

Sheen J (1990). Metabolic repression of transcription in higher plants. Plant Cell 2: 1027-1038.

Shi J, Kim KN, Ritz O, Albrecht V, et al. (1999). Novel protein kinases associated with calcineurin B-like calcium sensors in Arabidopsis. Plant Cell 11: 2393-2405.

Shin DH, Choi M, Kim K, Bang G, et al. (2013). HY5 regulates anthocyanin biosynthesis by inducing the transcriptional activation of the MYB75/PAP1 transcription factor in Arabidopsis. FEBS Lett. 587: 1543-1547.

Smeekens S (2000). Sugar-induced signal transduction in plants. Annu. Rev. Plant Physiol. Plant Mol. Biol. 51: 49-81.

Staswick PE, Serban B, Rowe M, Tiryaki I, et al. (2005). Characterization of an Arabidopsis enzyme family that conjugates amino acids to indole-3-acetic acid. Plant Cell 17: 616-627.

Touraine B, Muller B and Grignon C (1992). Effect of phloem-translocated malate on NO(3) uptake by roots of intact soybean plants. Plant Physiol. 99: 1118-1123.

Weinl S and Kudla J (2009). The CBL-CIPK $\mathrm{Ca}^{2+}$-decoding signaling network: function and perspectives. New Phytol. 184: 517-528.

Xu J, Li HD, Chen LQ, Wang Y, et al. (2006). A protein kinase, interacting with two calcineurin B-like proteins, regulates $\mathrm{K}+$ transporter AKT1 in Arabidopsis. Cell 125: 1347-1360.

Yan J, Niu F, Liu WZ, Zhang H, et al. (2014). Arabidopsis CIPK14 positively regulates glucose response. Biochem. Biophys. Res. Commun. 450: 1679-1683.

Yu SM, Lee YC, Fang SC, Chan MT, et al. (1996). Sugars act as signal molecules and osmotica to regulate the expression of alpha-amylase genes and metabolic activities in germinating cereal grains. Plant Mol. Biol. 30: 1277-1289. 